\title{
ILCEA
}

Revue de l'Institut des langues et cultures

d'Europe, Amérique, Afrique, Asie et Australie

37 | 2019

Des genres en Méditerranée : pratiques,

représentations et transfert

\section{Conteur méditerranéen : entre genre et médiation}

Mediterranean Storyteller: Between Gender and Mediation

Souad Jadli et Khadija Mouzon

\section{(2) OpenEdition}

Journals

Édition électronique

URL : http://journals.openedition.org/ilcea/7711

DOI : 10.4000/ilcea.7711

ISSN : 2101-0609

Éditeur

UGA Éditions/Université Grenoble Alpes

Édition imprimée

ISBN : 978-2-37747-099-0

ISSN : 1639-6073

Référence électronique

Souad Jadli et Khadija Mouzon, « Conteur méditerranéen : entre genre et médiation », ILCEA [En ligne], 37 | 2019, mis en ligne le 04 novembre 2019, consulté le 15 janvier 2020. URL : http://

journals.openedition.org/ilcea/7711 ; DOI : 10.4000/ilcea.7711

Ce document a été généré automatiquement le 15 janvier 2020

(C) ILCEA 


\title{
Conteur méditerranéen : entre genre et médiation
}

\author{
Mediterranean Storyteller: Between Gender and Mediation
}

Souad Jadli et Khadija Mouzon

\section{Introduction}

1 Le conte populaire constitue l'un des aspects les plus connus de la tradition orale dans plusieurs pays méditerranéens notamment la France et le Maroc ; il permet au conteur de transporter son public dans un monde imaginaire : «Le conte, comme le rêve, est toujours un trajet vers un autre monde, celui du "il était une fois" et du mensonge annoncé. » (Jean Derive, 2000 : 2)

2 Pour ce faire, le conteur doit satisfaire les différents goûts de son public hétérogène : tranches d'âge, identité culturelle et catégories sociales totalement différentes. La pérennisation du conte à travers le temps, est fortement liée à la maîtrise du conteur de plusieurs compétences stylistiques et langagières, afin d'assurer les multiples fonctions du conte : « Le conte est donc, comme dans la société traditionnelle, autant un outil de divertissement qu'une méthode d'éducation et de formation» (Milebou Ndjave, 2013 : 156).

3 Le conteur devient alors un médiateur entre le conte et le public : il assure le transfert du message, de la morale au public, et mesure la réactivité de ce dernier dans les différents modes ${ }^{1}$.

4 La pratique de l'art du conte, en France et au Maroc, ne se fait pas de la même manière ; nombreux sont les points de divergences, à titre d'exemple et non d'exhaustivité : le rôle et la place des conteuses femmes dans la transmission du conte; et le degré d'exploitation des outils du web social, plus précisément Facebook et YouTube dans la transmission et le maintien de cet art face à l'imprégnation de la société méditerranéenne par la haute technicité et le visuel. 
5 Ainsi, plusieurs questions nous préoccupent:

- Comment les conteurs (ses) marocains (es) et français (es) assurent-ils (elles) le rôle de la médiation? Dans quelles conditions?

- Quelle place occupent les conteuses femmes dans la pratique de l'art du conte?

- Comment les conteurs marocains et français exploitent-ils les outils du web social ?

Dans cet article, nous visons à répondre à ces questions en adoptant la structure suivante : la première section présente le rôle de médiation que joue le conteur ainsi que la place et le rôle des conteuses femmes dans la transmission de cet art. Dans la deuxième section, nous présenterons les composantes du web social et les avantages qu'il propose dans la redynamisation de ce métier. Pour illustrer notre approche, nous présenterons dans la troisième et dernière section une étude réalisée sur un échantillon de vidéos de conteurs français et marocains publiées à l'aide des outils du web social. En conclusion, nous ferons le point sur le bilan du travail effectué.

\section{Médiation et genre dans la pratique du conte}

7 Le conteur méditerranéen, en l'occurrence français et marocain, joue le rôle d'un médiateur entre le conte et le public qui le réceptionne, il a le don naturel ou acquis de rentrer dans l'histoire, de faire corps avec le verbe: "Le conteur est un créateur spontané. Il a une dimension symbolique. C'est un artiste. Il doit capter l'attention de son auditoire, susciter l'intérêt de ceux qui l'écoutent. Il doit faire entrer le public dans le monde qu'il crée, le temps du conte. » (Jala, 2006 :7)

8 Il puise dans son répertoire pour maintenir le suspense de son histoire jusqu'à la fin. Il apporte toujours des improvisations qui rendent le conte nouveau à chaque fois. L'art du conteur consiste aussi à introduire des variantes, et à ajouter des descriptions supplémentaires à une action donnée ou à un personnage: "En effet, celui-ci [le conteur] ne se contente pas de livrer simplement son récit, il le rythme de répétitions qui vont permettre la mémorisation, il l'agrémente de quantité d'adjectifs, l'interrompt soudainement pour solliciter une attention accrue, taquiner un auditeur ou attendre une question qu'il aura suggérée. » (Legey, $2010: 8$ )

9 Seul maître de son espace, il peut même marquer son histoire, de temps à autre, par un silence voulu, pour laisser éclore l'imaginaire de son public: "souvent, les traits les plus importants étaient suivis non d'explications, mais de silences et le conteur indiquait que, dans une histoire, aucune allusion n'est gratuite et que tout doit signifier quelque chose. » (Scelles-Millie, 1970 : 11)

10 Ainsi, les conteurs arrivent à créer un monde autour d'eux, de leurs histoires et de leurs visions de la vie: "Par la grâce d'un langage pas clair, éclaté sur lui-même, nos conteurs originels devaient réinventer un autre monde, donner des élans sans mots d'ordre, ouvrir les pays dessous les terres à cannes, les désigner sans les montrer et les laisser agir sans prescription en nous... un minutieux et très savant détour.» (Chamoiseau, 2016:24)

11 Afin d'assurer la médiation entre le conte et le public, le conteur / médiateur doit employer plusieurs techniques et procédés, il doit être en mesure de capter l'attention de son public : «Les conteurs par des répétitions, des accompagnements sur le guembri ou le tambourin, des chants intercalés, une mimique expressive, des interpellations et 
des invectives directes pour tenir l'auditoire en suspens, transforment le conte en un véritable scénario où le public joue son rôle. » $(2010: 14)$

L'emploi de tous ces moyens permettra le maintien du suspense de son auditoire, l'assurance que l'auditoire suit le fil des évènements de l'histoire et les actions des personnages.

Par ailleurs, la pratique de l'art du conte au Maroc et en France connaît plusieurs divergences :

- En France, les efforts se focalisent pour maintenir cet art en lui accordant un intérêt académique $^{2}$, de recherche ${ }^{3}$ et institutionnel à travers l'organisation de plusieurs manifestations culturelles autour du conte. Il est à noter également que la pratique de cet art connaît une très forte présence féminine. En effet, un grand nombre de conteuses françaises, de naissance ou de nationalité, pratiquent l'art du conte de plus en plus.

- Au Maroc, l'intérêt porté au conte s'est développé : «Plusieurs manifestations de contage sont relevées, compte tenu de l'engouement du public, à la fois jeune et adulte, qui retrouve ses repères identitaires avec ce genre de représentation ludique et symbolique : Association de la Maison du Conte du Maroc, Festival de conte de Rabat, Projet de contage pour valoriser le patrimoine oral de la région »(Chadli, 2016 :16).

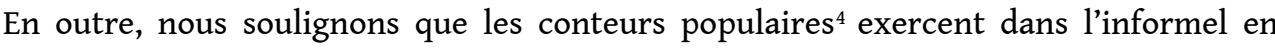
dehors de toute organisation institutionnelle, dans des places publiques. C'est notamment le cas de celle de la place Jemaa El-Fna déclarée par l'UNESCO ${ }^{5}$ patrimoine culturel immatériel de l'humanité.

Par ailleurs, les conteuses femmes pratiquaient initialement dans leurs foyers : «Celui [plaisir] de voir l'émulation agir sur nos mères, devenues conteuses pour un public plus nombreux qu'ordinaire, devenues conteuses publiques pour un soir. » (Djebel, $2011: 8$ )

Le champ de la pratique de cet art s'est timidement élargi: des conteuses professionnelles (comme Hafida Hamdane, Khadija Hassala et bien d'autres) qui animent des spectacles autour du conte dans différentes villes marocaines dans le cadre de manifestations culturelles.

Aujourd'hui, les changements sociaux, économiques et culturels, associés à une plus grande diversité des moyens modernes de transmission des savoirs, participent à une marginalisation progressive du spectacle de lhalqa ${ }^{6}$, autrefois seul moyen de distraction, les initiations auprès des anciens (au conte, au tambour ou à toute autre tradition) sont devenues rares. Par conséquent, l'activité professionnelle des conteurs risque de disparaître, d'où l'intérêt de rechercher d'autres alternatives et de conquérir d'autres horizons, comme les réseaux sociaux.

18 Dans la section suivante, il sera question de définir le web social et de présenter les avantages de certains de ses outils dans l'enrichissement du travail des conteurs méditerranéens.

\section{L'émergence du web social au service de la promotion du travail du conteur}

L'expression «web social» ou «web communautaire» (ex: YouTube, Facebook, Twitter, Snapchat, etc.) qualifie le franchissement d'une étape dans la participation innovante et dans la formation des communautés de création. Grâce à des techniques 
de développement nouvelles, chaque internaute se voit donner la possibilité d'être à la fois lecteur et contributeur : «le Web social révolutionne le monde à partir de 2004. Les réseaux sociaux ont une puissance incontournable et très évolutive avec 900 millions d'utilisateurs pour Facebook, 555 millions pour Twitter. Ils touchent aujourd'hui 1,2 milliard de personne. Un chiffre extravagant qui donne du vertige. La radio avait mis 38 ans pour toucher 50 millions d'utilisateurs, la TV 13 ans, le PC 4 ans, Facebook 2 ans et Google+ seulement... 88 jours! » (Digout \& Besson, $2016: 6$ ). La clé du succès, dans cette nouvelle étape de l'évolution du web, réside essentiellement dans l'intelligence collective et la collaboration.

Ainsi, avec les moyens offerts par ce web, le conteur méditerranéen jouit d'une nouvelle scène de communication virtuelle qui permet le contact inconditionné avec un public hétérogène possédant différentes caractéristiques socio-culturelles (âge, sexe, niveau social, niveau d'études, etc.). Il s'agit de substituer l'espace actuel par un espace virtuel, les critères de valorisation du conteur passeront donc de l'admiration par l'applaudissement, de la grandeur du cercle de public, au nombre de mentions «j'aime » ou le nombre croissant de partage du même conte sur la toile numérique.

Le contact qui était auparavant un contact direct synchrone se transformera alors en un contact indirect en mode asynchrone. Le conteur ne sera pas en possibilité de voir son public, de mesurer sa réactivité, comme nous l'avons déjà mentionné, mais plutôt il sera attaché à ses outils de communication divers (PC, portable...), pour suivre à chaque instant le devenir de son conte: Est-ce que son conte a récolté assez de mentions «j'aime» ou "partage » pour être populaire dans le monde virtuel? Est-ce que les commentaires sont positifs ? La manière de présentation du conte est-elle appréciée ou pas? Plusieurs questions auxquelles le conteur doit répondre pour pouvoir maintenir et réserver sa place dans un monde évolutif et impitoyablement concurrentiel.

Pourtant, tout ce que nous avons présenté jusque-là, nécessite avant tout une volonté $\mathrm{du}$ conteur pour utiliser ces plateformes, et un accompagnement des institutions culturelles pour rendre l'espace virtuel un nouvel espace de promotion de l'art du conte.

Dans la section suivante, nous présentons une étude faite sur un échantillon de vidéos de conteurs français et marocains.

\section{Conteurs méditerranéens au sein du web social}

S'inspirant de la réussite des outils du web social dans la transmission et le maintien du contage, nombreux sont les conteurs français ${ }^{7}$ qui ont su utiliser ces moyens au profit de leur art. De manière pensée, nous avons sélectionné quatre vidéos de quatre conteurs français (dont deux conteuses) en pleine scène, postées sur YouTube et nous avons analysé le degré de leur réception à travers l'analyse des données offertes par cette plate-forme. Le tableau ci-dessous illustre les résultats obtenus :

\begin{tabular}{|l|l|l|}
\hline $\begin{array}{l}\text { Conteur/ } \\
\text { conteuse }\end{array}$ & Données sur la vidéo & Nature du commentaire \\
\hline
\end{tabular}




\begin{tabular}{|c|c|c|}
\hline $\begin{array}{l}\text { Catherine } \\
\text { Zarcate }\end{array}$ & $\begin{array}{l}\text { Titre : Paroles de conteurs } \\
\text { URL : <https://www.YouTube.com/ } \\
\text { watch?v=IcUgBXaHN1U\&t=10s> } \\
\text { Date de mise en ligne : } 3 \text { déc. } 2009 \\
\text { Nombre de vue : } 68095 \\
\text { Nombre de «j'aime » }: 218 \\
\text { Nombre de «j'aime pas » : } 16 \\
\text { Nombre de commentaire : } 32\end{array}$ & $\begin{array}{l}\text { Les commentaires sont tous positifs, les } \\
\text { internautes expriment leur fascination face à } \\
\text { la qualité de la prestation de la conteuse. }\end{array}$ \\
\hline $\begin{array}{l}\text { Praline Gay- } \\
\text { Para }\end{array}$ & $\begin{array}{l}\text { Titre : Paroles de conteurs } \\
\text { URL : <https://www.YouTube.com/ } \\
\text { watch?v=pvQ4511kyGk> } \\
\text { Date de mise en ligne : } 3 \text { déc. } 2009 \\
\text { Nombre de vue : } 21817 \\
\text { Nombre de «j'aime » }: 49 \\
\text { Nombre de «j'aime pas » }: 2 \\
\text { Nombre de commentaire }: 1\end{array}$ & $\begin{array}{l}\text { Un seul commentaire où nous avons le mot } \\
\text { «merci » ce que nous pouvons qualifier de } \\
\text { positif. }\end{array}$ \\
\hline $\begin{array}{l}\text { Jean-Louis } \\
\text { Le Craver }\end{array}$ & $\begin{array}{l}\text { Titre : L'âne et le quincaillier par } \\
\text { Jean-Louis Le Craver } \\
\text { URL: <https://www.YouTube.com/ } \\
\text { watch?v=z657iUaRx24> } \\
\text { Date de mise en ligne : } 26 \text { sept. } 2012 \\
\text { Nombre de vue : } 5311 \\
\text { Nombre de «j'aime » }: 13 \\
\text { Nombre de «j'aime pas »: } 1 \\
\text { Nombre de commentaire : } 1\end{array}$ & $\begin{array}{l}\text { Le seul commentaire témoigne de } \\
\text { l'enchantement de l'internaute de l'histoire } \\
\text { contée. }\end{array}$ \\
\hline $\begin{array}{l}\text { Alain } \\
\text { Gaussel }\end{array}$ & $\begin{array}{l}\text { Titre : Paroles de conteurs } \\
\text { URL : <https://www.YouTube.com/ } \\
\text { watch?v=0jk_BO6t7lU> } \\
\text { Date de mise en ligne : } 8 \text { juil. } 2010 \\
\text { Nombre de vue : } 22304 \\
\text { Nombre de «j'aime » }: 83 \\
\text { Nombre de «j'aime pas » }: 4 \\
\text { Nombre de commentaire : } 5\end{array}$ & $\begin{array}{l}\text { Tous les commentaires sont positifs et } \\
\text { attestent la maîtrise du conteur. }\end{array}$ \\
\hline
\end{tabular}

Les informations recueillies sont à caractère positif, le nombre de vues a varié entre 5311 et 68095 vues, un chiffre important qui témoigne de l'utilité de cet outil pour toucher un plus grand nombre de public, les mentions « j'aime » dépassent largement les mentions «je n'aime pas»: nous avons un total de 363 mentions «j'aime» contre 27 mentions «j'aime pas». Par rapport aux commentaires, ils témoignent des compétences des conteurs dans la maîtrise de l'art du conte.

Face à la réussite des conteurs français en matière d'utilisation des outils du web social, notamment YouTube, nous avons mené une expérience qui consiste à sélectionner un 
échantillon de trois vidéos de conteurs marocains, dans des situations d'énonciation différentes, et de les mettre sur une chaîne YouTube et une page Facebook, toutes deux créées à cette fin. Nous visons, à travers cette expérience, d'une part, de sonder l'intérêt que peut porter éventuellement le public aux prestations des conteurs marocains avec ces nouvelles technologies, et mesurer, d'autre part, la réactivité virtuelle du public ou des internautes aux trois conteurs sélectionnés. Les lignes qui suivent décrivent la démarche adoptée. Dans ce qui suit, nous présentons les résultats obtenus.

\subsection{Sélection de l'échantillon}

La première étape de notre démarche a consisté à sélectionner l'échantillon à exploiter. Nous avons focalisé nos efforts à rechercher des vidéos sur la toile numérique de conteurs en représentation. Nous avons occulté la possibilité d'utiliser les données relatives à ces vidéos déjà en ligne pour la simple raison que nous voulons les traiter de telle sorte à respecter les critères suivants :

- Avoir des capsules de vidéos ne dépassant pas trois minutes ;

- La qualité technique de la vidéo (qualité de l'image, clarté de la voix et la stabilité de la scène) ;

- Le langage utilisé est un langage clair, ne contenant pas des mots vulgaires.

Cette sélection nous a permis de retenir les vidéos suivantes (Tableau 1) que nous avons classées par ordre de publication, sur la page Facebook et la chaîne YouTube.

\begin{tabular}{|l|l|l|}
\hline$n^{\circ}$ & Conteur & Description de la situation \\
\hline 1 & $\begin{array}{l}\text { Mohammed Barisse : âgé d'une cinquantaine } \\
\text { d'années. }\end{array}$ & $\begin{array}{l}\text { Durée : 2 min. 48 } \\
\text { Public : petits enfants } \\
\text { Lieu : espace fermé, maison } \\
\text { Description : le conteur raconte une } \\
\text { histoire populaire à propos d'un } \\
\text { personnage traditionnel nommé Hdidān } \\
\text { (malin) }{ }^{8} .\end{array}$ \\
\hline 2 & $\begin{array}{l}\text { Deux conteurs (le père et le fils) : le premier est } \\
\text { âgé d'une cinquantaine d'années, le fils est en } \\
\text { train d'apprendre le métier de son père. }\end{array}$ & $\begin{array}{l}\text { Dublée : 2 min. 20 } \\
\text { Espace : Place Aassarag à Taroudant } \\
\text { Description : les deux conteurs racontent } \\
\text { un conte traditionnel sur la confiance. }\end{array}$ \\
\hline 3 & $\begin{array}{l}\text { Deux conteurs nommés Mwalin Lehmam } \\
\text { (propriétaires des pigeons) en train de réciter de } \\
\text { la poésie populaire parlée et chantée (al-zajal) }\end{array}$ & $\begin{array}{l}\text { Durée : 2 min. 19 } \\
\text { Public : varié } \\
\text { Lieu : Place Jemaa El-Fna à Marrakech }\end{array}$ \\
\hline
\end{tabular}

Tableau 1 : Description de l'échantillon. 


\subsection{Création de la page Facebook et de la chaine YouTube}

Après avoir déterminé notre échantillon, nous avons entamé la deuxième étape de notre démarche qui consiste en la création des espaces virtuels, notamment une page Facebook et une chaîne YouTube que nous présentons respectivement.

1. La page Facebook: "Réception orale des contes populaires ${ }^{9}$; dans cette page (Figure 2) nous avons publié certaines images représentant les différents outils du web communautaire, et des vidéos de conteurs en pleine scène de deux manières différentes :

- La vidéo de Mohammed Barisse est hébergée directement dans Facebook ;

- La vidéo des deux conteurs sur la Place Assarag, et celle de Mwalin Lehmam, sont publiées à travers un lien YouTube.

31 Cette différence de mode de publication a pour objectif de connaître la méthode qui affecte le plus grand nombre d'internautes.

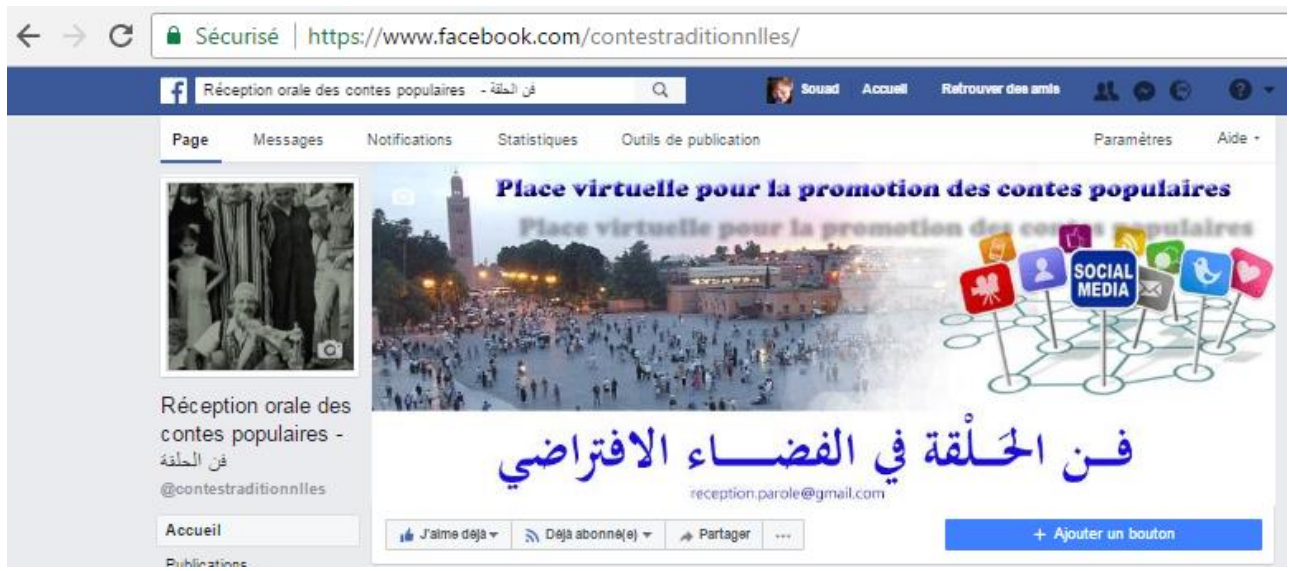

Figure 1: Page Facebook réception orale des contes populaires

La page Facebook a été créée le 22 Avril 2017, elle compte, à l'heure actuelle ${ }^{10}$, 117 abonnés dont les caractéristiques sociodémographiques sont les suivantes (Figure 3): nous avons une majorité d'hommes (97\% contre $3 \%$ de femmes) et une majorité de jeunes internautes : la tranche d'âge la plus représentée étant celle des 13 à 24 ans, suivie des 25 à 34 ans.

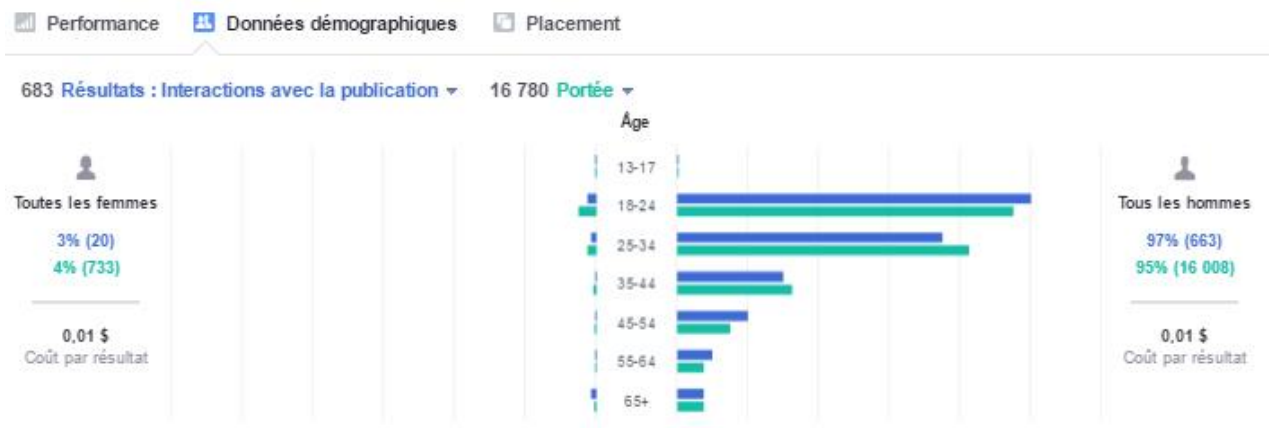

Figure 2 : Caractéristiques sociodémographiques du public virtuel

Ils constituent, en plus des internautes qui vont visiter la page, notre public virtuel.

2. La chaîne YouTube "Oralité et diversité ${ }^{11}$ : cette chaîne thématique pour la promotion de la tradition orale, notamment les contes populaires, a été créée 
le 4 février 2017, à partir de l'adresse Gmail reception.parole@gmail.com. La figure cidessous illustre la page d'accueil de la chaine « Oralité diversité » (Figure 3).

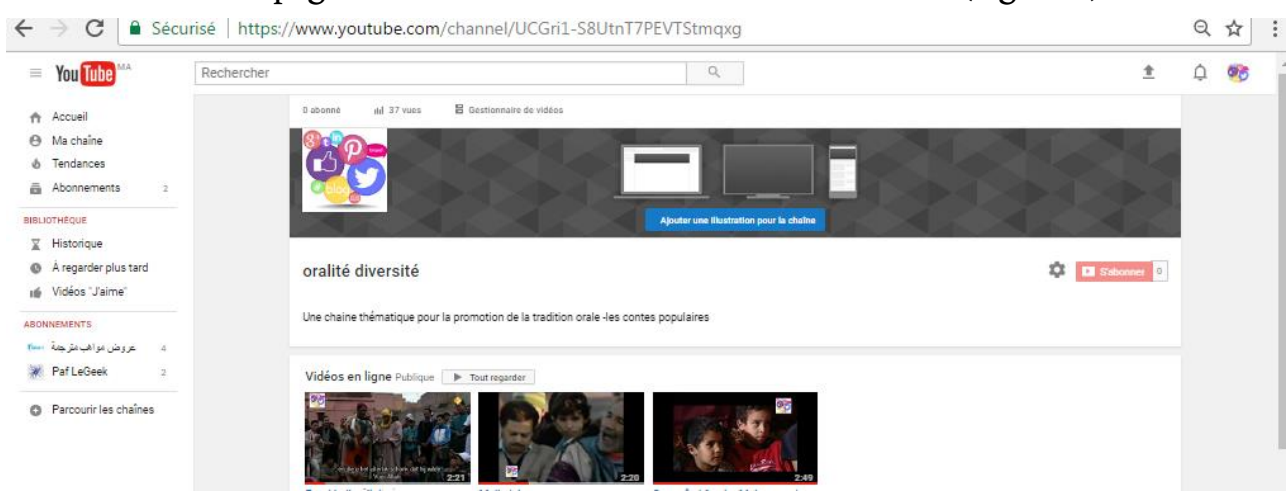

Figure 3: Chaîne YouTube « Oralité diversité ».

\subsection{Résultats de l'expérience de promotion de trois vidéos de conteurs}

Après avoir utilisé les outils du web communautaire, arrive l'étape de l'exploitation des données collectées. Ces données vont nous permettre d'avoir une idée sur le degré de réceptivité du conte populaire sur les réseaux sociaux. Nous commençons par l'analyse des données de la page Facebook que décrivent les graphiques de la figure ci-dessous :

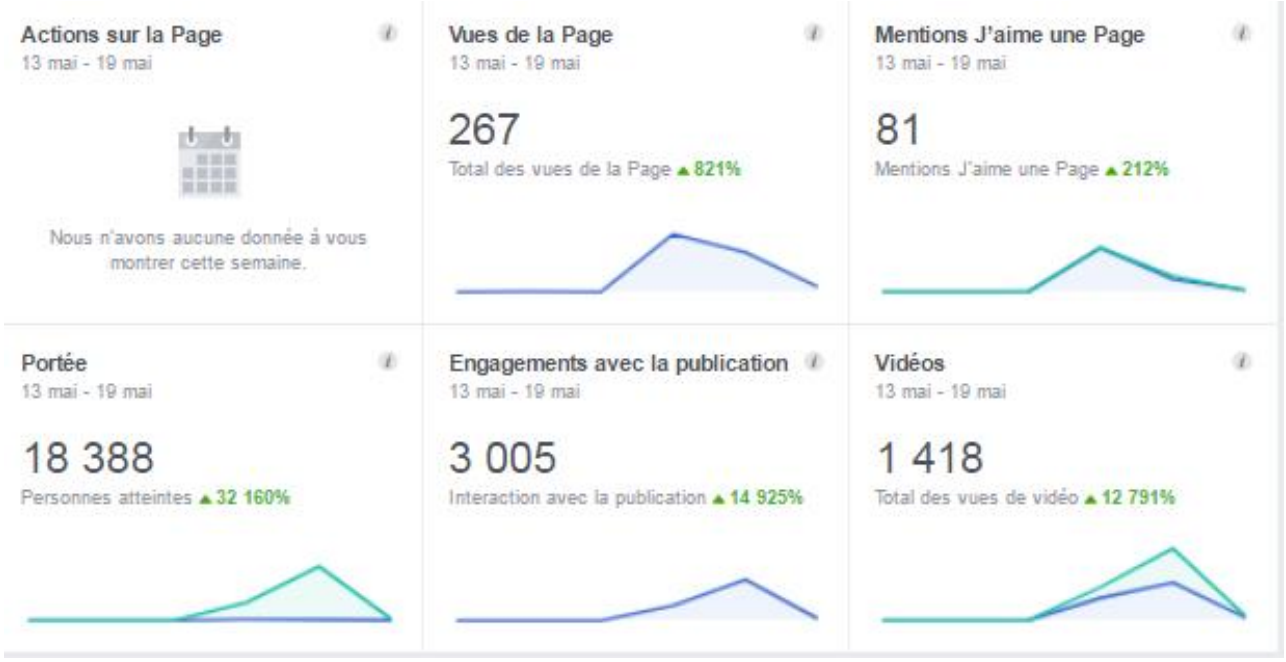

Figure 4 : Statistiques de la page Facebook.

Notre page a pu atteindre 18388 fans, un chiffre considérable qui témoigne de l'efficacité de cet outil ; mais le plus marquant est le nombre de vues des trois vidéos qui a atteint 1418 vues au bout d'une seule semaine ( du 13 au 19 mai 2017), ceci est dû essentiellement au partage des vidéos par les internautes qui ont visité la page, et apprécié ${ }^{12}$ le contenu des vidéos; ce qui a créé une inter connectivité virtuelle palpable à travers les mentions «j'aime » et " partager ». Or, il est à noter que les trois vidéos n'ont pas été perçues de la même manière. En se référant toujours aux statistiques offertes par la plateforme Facebook, nous avons noté que seule la première vidéo, celle du conteur Mohammed Barisse a pu récolter un total de 767 vues (Figure 5) durant la même période, soit $54,09 \%$ du total des vues, cela est dû peut-être à plusieurs critères, à savoir : 
- La méthode de publication de la vidéo : l'accès à la vidéo à partir de la page Facebook (pas besoin de passer à travers un lien) ;

- La simplicité du langage utilisé par le conteur ;

- La présence des enfants dans la scène, ce qui a évoqué probablement chez eux un retour aux contes des grands-mères.

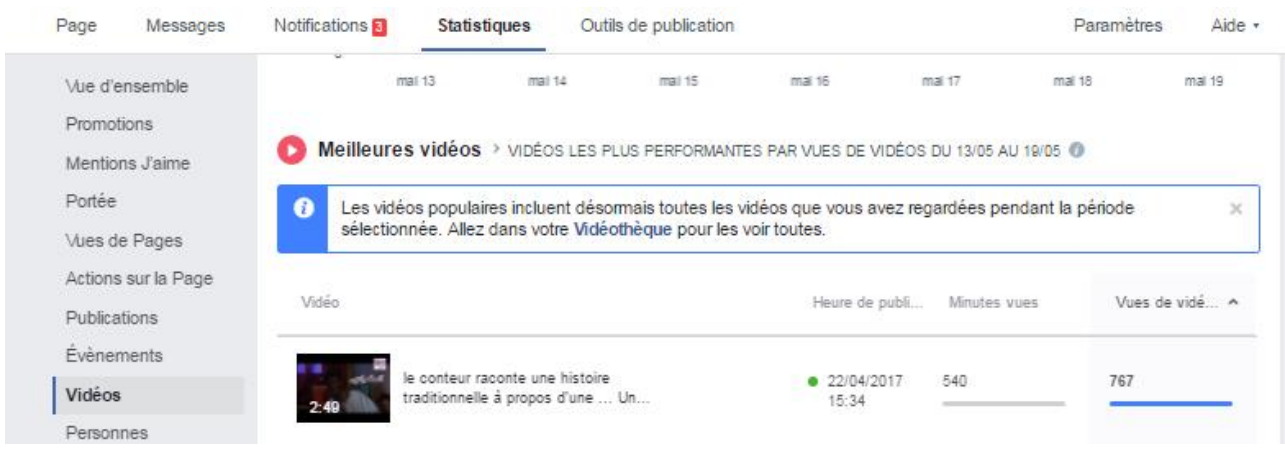

Figure 5 : Nombre de vues de la vidéo du conteur Mohamed Barisse.

Cela témoigne de l'efficacité de cet outil qui a permis la diffusion de la vidéo sélectionnée auprès d'un grand nombre de ce nouveau public virtuel, qui a des spécifications particulières; même à distance, ce public a pu apprécier cette tradition orale, et a réagi derrière les écrans de l'ordinateur ou avec les smartphones. Au lieu d'entendre les applaudissements, ou de mesurer la grandeur du cercle autour du conteur, nous avons compté le nombre des vues, et des partages du conte. Donc, ce nouveau public a pu réagir en un mode asynchrone : les internautes n'ont pas visualisé la vidéo au même moment, certes, mais ils l'ont appréciée.

Concernant les deux autres vidéos, elles ont eu un nombre de vues de 44 pour la deuxième vidéo, et 77 pour la vidéo de Mwalin Leffmam.

Quant aux données collectées à partir de la chaine YouTube "Oralité diversité », elles nous ont permis de mesurer le degré de réceptivité, comme le montre la figure cidessous :

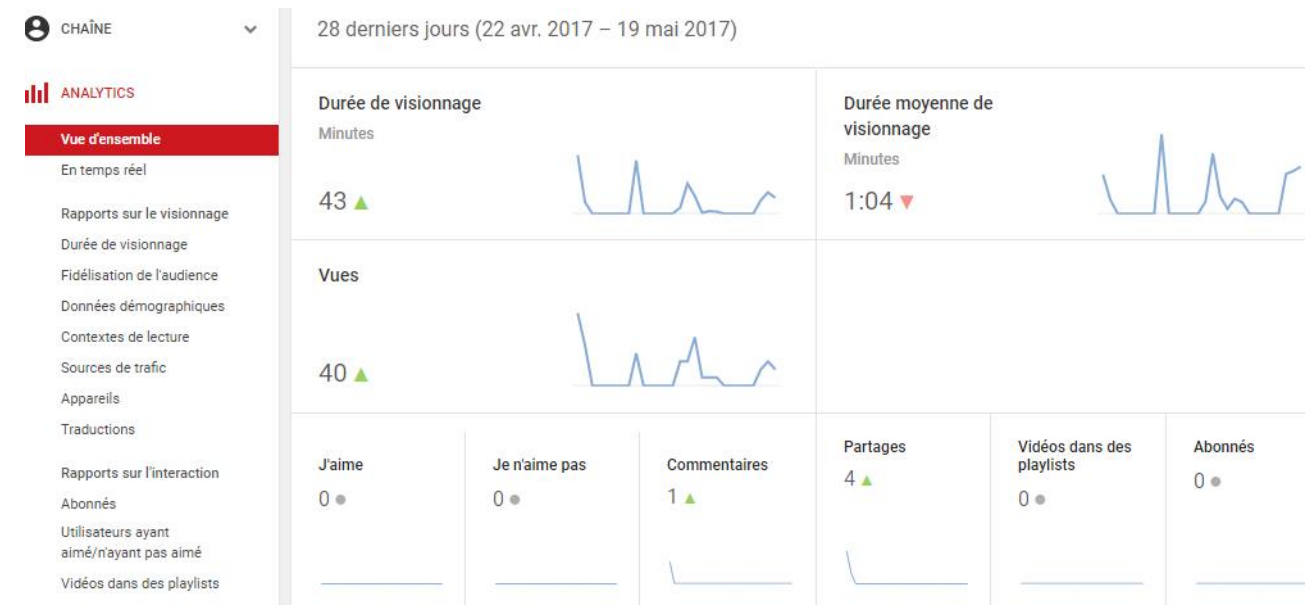

Figure 6 : Total de vues des vidéos.

Le total des vues des trois vidéos n'a pas dépassé 40 vues durant 28 jours ${ }^{13}$, un chiffre faible par rapport à la période écoulée, la réceptivité n'a pas été à la hauteur de nos espérances. Dans la situation ordinaire, le conteur peut parfaitement dépasser ce chiffre durant une telle période. Tout comme ce que nous avons constaté dans l'analyse 
de données de Facebook, la première vidéo de Mohammed Barisse a pu récolter, à elle seule, 21 vues soit $53 \%$ du total des vues (Figure 7), cela rejoint ce qui a déjà été dit sur l'appréciation du public de celle-ci.

Figure 7 : Statistiques de la chaîne YouTube.

42 Nous avons également noté que la réception de ces vidéos a dépassé le territoire du Maroc, pour atteindre le Canada (2,2 \%) et la France (1,1\%) (Figure 9), la faiblesse de ce pourcentage n'écarte pas la possibilité de bénéficier de cet outil dans la diffusion de notre tradition orale qui peut devenir plus populaire qu'elle ne l'est déjà.

\section{Top des régions}

\section{Durée de visionnage}

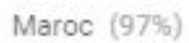

Canada $(2,4 \%)$

France $(1,1 \%)$

Russie $(0,0 \%)$

Figure 8 : Diffusion des vidéos sur la chaîne YouTube.

En comparaison des deux outils, nous soulignons que la plateforme Facebook est plus socialement efficace dans la promotion et le partage de la tradition orale, notamment les contes populaires, cela est probablement dû au nombre d'utilisateurs organisés sous forme de groupes contenant des milliers de membres qui peuvent partager à leur tour sur leurs propres comptes. Ceci crée une inter-connectivité virtuelle et un immense espace d'échange. Avec l'effet boule de neige, les vidéos des conteurs marocains peuvent arriver à des records en un laps de temps.

\section{Conclusion}

À travers cet article, nous avons essayé de démontrer la médiation que peut jouer le conteur méditerranéen. Il a également été question de la pratique féminine de cet art, encore timide au Maroc, ainsi que de l'utilité des outils du web social comme Facebook et YouTube dans la reconnaissance sociale du rôle du conteur méditerranéen dans la promotion de l'art du conte. Nous avons travaillé sur un échantillon de sept ${ }^{14}$ vidéos au total ; par la suite nous avons mesuré le degré de réceptivité du public virtuel à travers les statistiques offertes par ces outils. Les résultats sont prometteurs, surtout par rapport aux conteurs marocains qui ne font qu'une utilisation timide de ces outils. Même si les aires géographiques sont différentes, le plaisir lui est commun : le fait de découvrir l'imaginaire à travers les paroles d'un conteur. 


\section{BIBLIOGRAPHIE}

CHADLI El-Mostafa (2015), « Quelles approches pour une valorisation du patrimoine immatériel de la région au Maroc ?», La Revue des Arts de l'Oralité (5, Printemps 2016), Béni-Mellal, Maroc : Association OCADD.

Chamoiseau Patrick (2016), Matière de l'absence, Paris : Éditions du Seuil.

DERIVE Jean (2000), « Nicole Belmont, Poétique du conte. Essai sur le conte de tradition orale. Paris, NRF/Gallimard, 1999 », Études rurales (155-156), <http://journals.openedition.org/etudesrurales/ 65>.

Digout Jacques \& BESSON Laurent (2016), Le Web 2.0 au service de la création de valeur, Paris : Éditions Vuibert.

DJEBEL Mourad (2011), Contes des trois rives, Paris : Éditions Actes Sud.

JALA (2006), Majolay ou l'art du conte créole, Case-Pilote, Martinique : Éditions Lafontaine.

LEGEY Doctoresse (2010), Contes et légendes populaires du Maroc, Casablanca, Maroc : Éditions du Sirocco.

LEGEY Doctoresse (2009), Essai de folklore marocain, Casablanca, Maroc : Éditions du Sirocco.

Milebou Ndavé \& KelLy Marlène (2013), « Pierre-Claver Akendengué et l'art de chanter le conte », U. Baumgardt \& J. Derive (dir.), Littérature africaine et oralité, Paris : Éditions Karthala.

MilleRAND Florence, ProulXs Serge \& RUEFF Julien (2010), Web social : mutation de la communication, Québec : Presses de l'université du Québec.

\section{NOTES}

1. Le mode synchrone dans le cas où le conteur a un contact direct avec son public; et le mode asynchrone lorsque le conteur utilise Facebook ou YouTube pour partager ses spectacles sous forme de vidéos.

2. Plusieurs formations sont proposées au profit des personnes souhaitant devenir des conteurs, ou au profit des conteurs qui souhaitent améliorer leurs performances.

3. On assiste depuis des années à l'augmentation de nombre de productions littéraires et sociologiques autour du conte.

4. Hakawātī ou hlayqī en dialecte marocain.

5. Cf. site web de l'organisation: < https://ich.unesco.org/fr/RL/lespace-culturel-de-la-placejemaa-el-fna-00014>.

6. L'art du spectacle marocain.

7. On compte plus de 600 conteurs professionnels français qui exercent en utilisant les outils du web social, chiffre donné par la conteuse française Catherine Zarcate qui fait partie des artistes à l'origine du renouveau du conte en France.

8. Hididān est un personnage rusé et doué d'une intelligence exceptionnelle. Il parvient à s'extirper des pires situations et à obtenir gain de cause grâce à son talent de manipulateur. Sa philosophie dans la vie constitue à faire le moins d'effort possible tout en usant de son intelligence pour s'en sortir.

9. <https://www.facebook.com/contestraditionnlles/>. 
10. La date du dernier relevé de chiffre correspond au 30 décembre 2018.

11. <https://www.YouTube.com/channel/UCGri1-S8UtnT7PEVTStmqxg>.

12. Même si le nombre des commentaires est très réduit ne dépassant pas quelques commentaires, nous avons jugé l'appréciation du public à travers le nombre important de vues.

13. Nous avons souligné une faible réactivité du public sur la chaîne YouTube, c'est la raison pour laquelle nous avons préféré sélectionner une période plus étendue que celle accordée à la page Facebook.

14. Quatre vidéos de conteurs français et trois vidéos de conteurs marocains.

\section{RÉSUMÉS}

Dans certains pays de la Méditerranée, précisément la France et le Maroc qui constituent notre terrain d'étude, le conte populaire constitue l'un des aspects les plus marquants de la tradition orale. En effet, le spectacle du conte permet la transmission de la tradition orale à travers les histoires racontées. Le conteur a comme fonction de satisfaire les différents goûts de son public hétérogène : tranches d'âge et catégories sociales totalement différentes. Or, la question du genre est fortement posée puisqu'il est observé que cette pratique culturelle est dominée par les conteurs hommes, avec une présence timide des conteuses femmes surtout au Maroc où les conteurs hommes sont omniprésents sur les places publiques. Un autre défi est levé pour le maintien de l'art du conte, c'est celui d'exploiter les outils du web social pour attirer un grand nombre de public, surtout que la société méditerranéenne est inondée par la technologie et le visuel. De ce fait, le conteur / la conteuse doit être en mesure de satisfaire les goûts de ce public virtuel à travers l'utilisation des outils du web social les plus populaires, notamment Facebook et YouTube.

L'objectif de ce papier est de répondre aux questions citées précédemment, d'analyser certaines vidéos de conteurs (ses) marocains (es) et français (ses) mises en ligne sur YouTube et Facebook et de mesurer la réactivité du public pour mettre en évidence la médiation que joue le conteur / la conteuse entre son spectacle et son public virtuel.

In some Mediterranean countries, precisely France and Morocco, which are our field of study, the popular tale is one of the most striking sides of oral traditions. Indeed, the storytelling show allows the transmission of oral traditions through the stories told. The storyteller's goal is to satisfy the different tastes of his heterogeneous audience that have very different age groups and social categories. However, an observer can deduct that the gender of the storyteller matters, because it is observed that male storytellers dominate this cultural practice with a timid presence of female storytellers especially in Morocco where male storytellers are omnipresent in public places.

Another challenge for maintaining the art of storytelling is the use of modern tools like the social media (Facebook and Twitter) and streaming websites (YouTube) to attract a larger audience in order to satisfy the tastes of his virtual audience, especially since Mediterranean society has known new technologies and new habits.

The objective of this paper is to answer the previously mentioned questions, to analyze some videos of Moroccan and French storytellers posted on YouTube and Facebook and to measure the responsiveness of the audience to highlight the mediation that the storyteller plays between his show and his virtual audience. 
INDEX

Keywords : storytelling, gender, web, society, Mediterranean, storyteller, tradition, mediation, taste

Mots-clés : conte, genre, web, société, Méditerranée, conteur, tradition, médiation, goût

\section{AUTEURS}

SOUAD JADLI

Université Hassan II - Casablanca, LALICO, Maroc

\section{KHADIJA MOUZON}

Université Hassan II - Casablanca, LALICO, Maroc 